\title{
Valuation of Wetland Attributes: An Application of Choice Experiment Approach: A Case Study of Lake Koka
}

\author{
Bulo Feyisa $^{1}$, Mekonnen Bersisa ${ }^{2}$ \\ ${ }^{1}$ Department of Economics, Jinka University, Jinka, Ethiopia \\ ${ }^{2}$ Department of Economics, Ambo University, Ambo, Ethiopia
}

Email address:

feyisabulo16@gmail.com (B. Feyisa),mbersisa@gmail.com (M. Bersisa)

\section{To cite this article:}

Bulo Feyisa, Mekonnen Bersisa. Valuation of Wetland Attributes: An Application of Choice Experiment Approach: A Case Study of Lake Koka. Journal of Investment and Management. Vol. 8, No. 5, 2019, pp. 76-85. doi: 10.11648/j.jim.20190805.11

Received: August 13, 2019; Accepted: September 11, 2019; Published: January 4, 2020

\begin{abstract}
Ethiopia has many lakes, the greater part of which is found in the Rift-valley, that are central for the development of economy of the country. One of these Lakes is Lake Koka, which is found inside the Ethiopian Rift Valley, and is an artificial lake built in 1960 to generate hydroelectric power. In spite of different advantages provided by Lake Koka, absence of powerful administration, uncontrolled irrigation, intensive agriculture and different human made activities has prompted to the degradation of the wetland. Human made activities such as, using wetland as waste disposal, over grazing, etc. aggravate the problems of the lake. To assign monetary values for the multi-functions and services offered by this Lake wetland ecosystem, this study applied choice experiment valuation method by identifying five attributes i.e. fish stock, biodiversity, water quality, availability of water and monetary payment. Multinomial logit, random parameter logit and latent class models were used to analyze the data collected from a sample of 200 respondents. The marginal willingness to pay for the improvement of water availability attribute is the highest which is birr 55 and it is significant at $1 \%$ level. Respondent's willingness to pay for an increase in the level of water quality of Lake Koka and biodiversity from current situation of the lake, other things being constant, is birr 42 and birr 12 annually respectively. Respondents gave more value to the availability of water than biodiversity and water quality attributes.
\end{abstract}

Keywords: Koka Lake, Choice Experiment, Multinomial Logit, Mixed Logit, Random Parameter Logit, Latent Class Model

\section{Background}

Wetlands are areas where water is the essential element for controlling the earth and the related plant and diverse biodiversity. It is surface of land that founds at or near to water body, or where the land is secured by shallow water, which depth is not more than 6 meters. They are the Earth's most beneficial eco-systems, which give diverse environmental functions and services, for example, flow and flood control, water quality upkeep, natural surroundings for plant and animal species, biodiversity, carbon sequestration, recreational services and other life-support services [18].

There are a wide range of sorts of wetland, for example, territories of bog and fen, marsh, costal and marine related. It may be regular or synthetic and it can be changeless or occasional. The water in wetlands can stream or static, and can be new, salty or saline [24]. However, numerous wetlands have been considered as wastelands and futile environment and they are degraded and drained.

To this day, many wetlands are under increasing pressure from human made activities, such as conversion of wetlands to intensive agricultural use and to other industrial and residential uses; their drainage as a result of excessive irrigation in agriculture; pollution as a result of chemical run-off from intensive agricultural production, and industry. Other factors considered to affect the management of wetlands include poverty and economic inequality, pressure from population growth, immigration and mass tourism, and social and cultural conflicts [18].

Since the total area of the world's wetland is certainly known it is difficult to quantify the lost area of wetland of the world. However, there are some figures for different countries that show the amount of lost wetland. These ecosystems are distributed throughout the world and estimated to cover 12.8 million $\mathrm{km}^{2}$ of the total area of the world [15] that covers $6.4 \%$ of the surface of the planet earth. In Africa the area of the wetland is estimated to 
vary between $1 \%$ and $16 \%$ or 220,000 and $1,250,000 \mathrm{~km}^{2}$ of the total area of the continent respectively.

Ethiopia has a wide range of wetlands aside from marine-related wetlands, since it is land locked country, and they are estimated to cover over $2 \%$ of the region [5]. However, different studies show that, they are under pressure both from natural and human made activities.

Ethiopia has many lakes, the greater part of which is found in the Rift-valley, that are central for the development of economy of the country. One of these Lakes is Lake Koka, which is found inside the Ethiopian Rift Valley, and is an artificial lake built in 1960 to generate hydroelectric power. From that time onward, it has been serving in parallel at least 15,000 residents around the Lake as source of water for drinking, cleaning, animal watering, irrigation, fishing, etc. [25] Lake Koka, with a surface area of $250 \mathrm{~km}^{2}$, is found at a distance of 50 miles south of the Capital of Ethiopia, Addis Ababa. The lake has been being used by various stakeholders with different level of power to control this asset. However, because of the current state of the lake, highly polluted, the residents are affected from many dimensions. They suffer from poor sanitation and water borne diseases, such as chronic diarrhea that are resulting from drinking the toxic water.

Even though it is difficult to value wetland, as there is no market for them and the nature of the ecological products, it is possible to value the goods and services obtained from the ecosystem. As wetland ecosystems could be a difficult task especially because of the complexity of the ecosystem and the requirement for multi-disciplinary services in the determination of its various components it is difficult to give value to wetlands [4] But environmental valuation methods are good enough to give valuable information about the ecosystem.

Therefore, the purpose of this study is to value the marginal willingness to pay for the improvement of the economy of Lake Koka, by using the recent and most appropriate method of environmental valuation method, a choice experiment method. The result of the study can be used as the starting point towards the solution to these serious problems to policy makers and government about the value of a Lake. Even though there are different techniques to estimate the environmental goods and services, in this study choice experiment valuation technique was used in order to estimate the economic value of multiple goods and services which are provided by Lake Koka wetland ecosystem.

This is because choice experiment method is the most appropriate and recent technique to estimate the multifunction and benefits provided by environmental goods and services. It combines random utility theory and characteristics theory of value to estimate the MWTP of the participants for different benefits of the environmental goods and services

\section{Objective of the Study}

\subsection{General Objective}

The general objective of this study is to measure the total economic contribution of Lake Koka wetland ecosystem to the welfare of the society.

\subsection{Specific Objectives}

1. To identify the factors that affects the Willingness to pay for different attributes of Lake Koka wetland.

2. To Estimate the marginal willingness to pay for each attributes of the wetland.

3. To estimate marginal rate of substitution between attributes of the Lake Koka.

4. To estimate the welfare impacts of improvement of each attributes of the wetland area.

\section{Literature Review}

Different scholars define wetland ecosystem in different ways, because there are different types of wetlands. But the most common broad definition for wetland resources is found in the Ramsar convention which was adopted in Iran in 1971. According to Ramsar convention More than 50 stakeholder interests biased definition and assumptions have been given to wetlands. But, the broadest and most international definition is as follows;

Wetlands are: "Areas of marsh, fen, and peat land, or water, whether natural or artificial, permanent or temporary, with water that is static or flowing, fresh, brackish or salt, including areas of marine water the depth of which at low tide does not exceed six meters".

The three major classifications of wetlands adopted by the Ramsar Convention are

Salt water: Marine, estuarine, lagoonal, saline inlands

Freshwater: Riverine, lacustrine, palustrine (marshes, etc.),

Manmade: Aquacultures, agricultures, pits (salt, rocks, coal, mineral, etc.), urban/industrial, reservoirs (hydro-dams, irrigation dams, etc.)

Wetlands are areas where water is the basic component for controlling the earth and the related plant and various biodiversity. It is surface of land that occurs at or closes water body, or where the land is secured by shallow water, which depth is not more than 6 meters. They are the Earth's most valuable eco-system, which give assorted ecological function and services, for instance, flow and flood control, water quality upkeep, regular surroundings for plant and animal species, biodiversity, carbon sequestration, recreational and other life-support services [18].

\section{Terminologies Related to Wetlands}

Attributes: These are aspects of wetlands which do not necessarily provide a function or support a use, but is valued by a group within society. In other words it relate to the structure and organization of biodiversity, such as beauty, rarity or diversity, and generate less tangible values such as spiritual, educational, cultural and recreational value.

Uses: The direct utilization of some aspect of wetlands. Economists recognize these as "Direct use values".

Goods: These are more like uses, above. For instance, they can be referred to as harvested resources, such as fish.

Services: These are more like functions, or they are processes that contribute to economic production or save costs, 
such as water purification.

[1] Categorized the services obtained from ecosystems as follows:

1. Provisioning services such as food and water;

2. Regulating services such as flood and disease control;

3. Cultural services such as spiritual, recreational, and cultural benefits; and

4. Supporting services, such as nutrient cycling, wastes assimilation, etc. that maintain the conditions for life on Earth.

Functions: Aspects of wetlands that potentially or actually support or protect human activities or human properties without being used directly, or protect natural systems or natural processes. These are also known as "Indirect use values" by economists. Benefits: These are commonly known as functions, uses, values, and attributes, features, goods and services. They are defined as any one of these terms which may have a value to people, wildlife, natural systems or natural processes.

\section{Methodology of the Study}

\subsection{Data Source}

The data sources for this paper are based on primary data collected from residents of the residing kebeles, using a stratified random sampling framework. The main sampling unit of the survey is the household. The choice experiment survey was then administered to the representative of the sample population in terms of income, social status, proximity to the wetland area.

\subsection{Survey Design}

The foundation for any stated preference experiment is a survey design. The manipulation of the levels of the variables does not occur in a random manner. Rather it needs a specialized form of statistics to determine what manipulations to make and when to make them [12]. There are four steps involved in the design of our choice experiment:

Step one: Definition of attributes and attribute levels

Attributes are determined based on local workers evidence and by taking into account respondents' characteristics and capacity to understand the attributes, which is farmers, in this case. On the bases of consultation with various experts and local residents around Lake Koka, five attributes including the payment attribute were determined. After attributes were identified and defined, the level of each attribute was determined. The levels of the attributes were selected in such a manner that it satisfies the characteristics of reliability and feasibility.

The attributes included in the study are, Fish stock, water quality, Biodiversity, availability of water and monetary payment.

\section{Step two: Experimental Design}

The next step after the relevant attributes and attribute levels were identified is construction of choice sets via experimental design. Choice sets were formed by using different levels of the attributes. The combination of different levels of attributes yields different alternative scenarios and then choice sets. In this study five attributes were used. Including status quo, quality of water has three levels, while the attributes of fish stock, Biodiversity, availability of water and monetary payment have four levels each. The number of wetland management scenarios that can be generated from five attributes, four with four levels and one with 3 levels is 768 (i.e. $4^{4} * 3^{1}=768$ ). This full factorial design may lead to very large combinations which could not be practicable and it is more than the respondents could be expected to cope with.

Step three: Questionnaire development

The questionnaire was classified in to three parts. First, respondents were asked questions related socioeconomic data (age, marital status, level of education, family size, proximity to the wetland, monthly household income and monthly expenditure) were presented to the respondents. The second part of the questionnaire consists of questions related to wetland and the respondents' observations about the Lake and its resources. In the last section the questionnaire consists of the choice experiment questions. The description about the general features of the Lake, its attribute and their levels, and about the payment vehicle were given to the respondents before directly enter into choice experiment. In addition to this, pictures that could explain the attribute levels in a simple ways were prepared since most of respondents are farmers.

Step four: Choice of Sample and Sampling strategy

The sample size for this study will be conducted based on stratified random sampling. Thus population is first divided into group that is mutually exclusive groups each representing a proportion of the total population. As discussed in [8] the basis for creating the groups can be any characteristic common to the population (e.g. age, income, location, gender etc.) with the exception of choice.

\subsection{Model Specification}

\subsubsection{Theoretical Framework}

In this study choice experiment method was employed. The $\mathrm{CE}$ technique is an application of the characteristics theory of value [16], combined with random utility theory $[11,27]$. It thus shares strong links with the random utility approach to value the environment using revealed preference data [28]. Random utility theory formulated [3,2] and consumer choice model formulated [28] are foundations for choice experiment. These foundations are used in estimating the effects of product attributes and individual characteristics and in computing willingness to pay indicators [21]. Thus, the total utility derived from consumption of a good is a function of the observable component whose value depends on the attributes of the good and the unobservable error term which is assumed to be independently and identically distributed [29] Respondents are asked to choose between different bundles of (environmental) goods, which are described in terms of their attributes, or characteristics, and the levels that these take.

\subsubsection{The Characteristics Theory of Value}

The choice experiment method has its theoretical grounding in model of consumer choice. Consumers derive satisfaction not from goods themselves but from the attributes they provide $[4$, 
7]. According to the characteristics theory of value, the probability of choosing a specific alternative is a function of the utility linked to the same alternative. Moreover, the utility derived from each alternative is assumed to be determined by the preferences over the levels of the attributes provided by that alternative [5].

The assumption that individuals derive utility from the characteristics of a good rather than from the good itself, implies that a change in one of the characteristics (such as the price) may result in a discrete switch from one good to another will however affect the probability of choosing that specific commodity on the margin

The representative individual is assumed to have a utility function of the form:

$$
\mathrm{Uin}=\mathrm{U}(\mathrm{Zin}, \mathrm{Sn})
$$

When for any individual $n$, a given level of utility will be associated with any alternative $i$. Alternative $i$ will be chosen over some other option $j$ if utility from alternative $i$ is greater than utility from alternative $j$. Utility derived from any option is assumed to depend on the attributes, $Z$, of that option (for example, water quality and the nature of the surrounding landscape). These attributes may be viewed differently by different agents, whose socioeconomic characteristics $\mathrm{S}$ will also affect utility. Assume also that the utility function can be partitioned into two parts; one deterministic which is observable, and one random and unobservable. Then Equation (1) can be re-written as:

$$
\mathrm{Uin}=\mathrm{V}(\mathrm{Zin}, \mathrm{Sn})+\varepsilon(\mathrm{Zin}, \mathrm{Sn})
$$

And the probability that individual $\mathrm{n}$ will choose option $i$ over other options $j$ is given by:

$$
\text { Prob }(i \mid \mathrm{C})=\operatorname{Prob}\{\text { Zin }+\varepsilon i n>Z j n+\varepsilon j n, \text { all } \mathrm{j} \in \mathrm{C}\}
$$

Where $\mathrm{C}$ is the complete choice set. In order to estimate Equation (3), assumptions must be made over the distributions of the error terms. The usual assumption made is that the errors are Gumbel-distributed (also known as the Type I Extreme Value distribution) and independently and identically distributed implies that, for any individual, the ratio of choice probabilities of any two alternatives is entirely unaffected by the systematic utilities of any other alternatives [11]. CE data sets can be tested for this independent from irrelevant alternatives (IIA) property, but if IIA is found to be violated, then the standard random utility model can no longer be applied.

Furthermore the systematic component of the utility function can be expressed by using vector of explanatory variables and their coefficients as follows.

$$
\mathrm{V}_{\text {in }}=\beta^{\prime} \mathrm{x}_{\text {in }}
$$

Equation (4) again can be used to write the probability that consumer $\mathrm{n}$ will choose option $\mathrm{i}$ in terms of systematic and error components which is used to estimate the values of vector of parameters $(\beta s)$ in the following way:

$$
\mathrm{P}(\mathrm{i} / \mathrm{Cn})=\mathrm{P}\left[\left(\beta^{\prime} \mathrm{x}_{i n}+\varepsilon_{\mathrm{in}}\right)>\mathrm{P}\left(\beta^{\prime} \mathrm{x}_{\mathrm{jn}}+\varepsilon_{\mathrm{jn}}\right)\right] \text {, for all } i \text { and } j \text { is an element of } \mathrm{C}
$$

By assuming that the consumers are maximizing their utility, they chooses option $i$ from option $j$ in the choice set $\mathrm{C}_{\mathrm{n}}$ if and only if the probability that the systematic and random components of option $i$ is greater than the systematic and random components of option $\mathrm{j}$.

\subsection{The Multinomial Logit Model}

\subsubsection{Basic MNL Model}

This model shows the importance of the attributes in explaining respondents' choices across different options in a choice set, a status quo and other alternatives with changes in attributes.

To estimate the choice probabilities using Multinomial Logit (MNL) model, it is assumed that the random components are independently and identically distributed (IID), with the implication that alternatives are independent from irrelevant attributes (IIA).

Given the assumption of the above IID Gumble distribution of the random component and independence between alternative scenarios and individual attributes, the probability of choosing alternative scenario in MNL equation has the following representations.

$$
\mathrm{P}(\mathrm{i})=\frac{\exp \lambda \beta x i}{\sum \exp \lambda \beta x j}
$$

Where, $\lambda$ is scale parameter in which true parameters are confounded with in it. It is not possible to identify this parameter from the data (Alpizar et al., 2003). The scale parameter thus has an impact of restriction in interpretation of estimated coefficient. Coefficient interpretation in MNL model is relative to the reference or base categories group. In MNL model the scale parameter has the inverse relationship with the variance of error term and is given by $\delta=\frac{\pi^{2}}{6 \lambda^{2}}$ (Adamowicz et al., 1998) and (Alpizar et al., 2003)

The model was specified in such a way that the probability of selecting a particular scenario alternative was a function of attributes of that scenario and of the alternative specific constant. Even though heterogeneity of preferences is likely to exist, the basic model is based on the assumption that preference of individuals is homogeneous. The indirect utility of basic model (model without the socio-demographic characteristics) from the proposed wetland improvement will take the following form:

$$
\mathrm{Vi}=\beta+\beta 1 f s t+\beta 2 b i o d+\beta 3 w q+\beta 4 a o w+\beta 5 p y n t
$$

Where $\mathrm{Vi}$ is the indirect utility for three alternatives (alternative 1, alternative 2 and status quo); $\beta$ refers to the alternative specific constant and $\beta 1, \beta 2, \beta 3, \beta 4$ and $\beta 5$ are coefficients associated with the attributes of fish stock (fst), biodiversity (biod), water quality (wq), availability of water (aow) and payment (p) or more specifically; 


$$
\begin{gathered}
\mathrm{V} 1=\mathrm{ASC} 1+\beta 1 f s t+\beta 2 \text { biod }+\beta 3 w q+\beta 4 \text { aow }+\beta 5 \text { pynt } \\
\mathrm{V} 2=\mathrm{ASC} 2+\beta 1 f s t+\beta 2 \text { biod }+\beta 3 w q+\beta 4 \text { aow }+\beta 5 \text { pynt } \\
\mathrm{V} 3=\beta 1 \text { fst }+\beta 2 \text { biod }+\beta 3 w q+\beta 4 \text { aow }+\beta 5 \text { pynt }
\end{gathered}
$$

Where ASC is the alternative specific constant that captures effect of any attribute (not included in the choice specific attributes) on utility.

V1 and V2 represent the indirect utilities derived from environmental improvements in the lake i.e. scenarios with options 1 and 2 .

$\mathrm{V} 3$ represents the indirect utility in the status quo option. It is to be noted that it is difficult to make any interpretation of the coefficients as they are confounded by a scale parameter i.e. they are dependent on the variance of the error involved in the estimation process. But it is possible to compare the sign and difference in magnitudes of the coefficients [14]. Moreover, ASC1 and ASC2 are considered to be equivalent.

\subsubsection{Extended MNL Model}

The basic multinomial logit model assume that preference of respondents are homogenous i.e. it assumed that preferences are homogeneous across individual respondents. Therefore, extended MNL model is estimated that relax this assumption i.e allow heterogeneity in preference across individual respondents. This can be done by allowing the interaction of socioeconomic variables with either attributes or ASCs in to the model. Hence, Seven socio-demographic variables age, sex, distance from the Lake, family size, income, education level and monthly expenditure, were included in the extended MNL model to estimate the effects of the variables on the probability of the respondent to choose either alternatives. The extended MNL model has the following form.

$$
\begin{aligned}
& \mathrm{V} 1= \mathrm{ASC} 1+\beta 1 f s t+\beta 2 \text { biod }+\beta 3 w q+\beta 4 a o w+\beta 5 p y n t+\beta 6 A S C A G E+\beta 7 A S C S E X \\
&+\beta 8 \text { ASCEDUC }+\beta 9 \text { ASCFMSZ }+\beta 10 \text { ASCDIST }+\beta 11 \text { ASCMEXP } \\
& \mathrm{V} 2=\mathrm{ASC} 2+\beta 1 f s t+\beta 2 \text { biod }+\beta 3 w q+\beta 4 a o w+\beta 5 p y n t+\beta 6 A S C A G E+\beta 7 A S C S E X \\
&+\beta 8 \text { ASCEDUC }+\beta 9 \text { ASCFMSZ }+\beta 10 \text { ASCDIST }+\beta 11 \text { ASCMEXP } \\
& \mathrm{V} 3= \beta 1 \text { fst }+\beta 2 \text { biod }+\beta 3 w q+\beta 4 \text { aow }+\beta 5 p y n t+\beta 6 \text { ASCAGE }+\beta 7 \text { ASCSEX }+\beta 8 \text { ASCEDUC } \\
&+ \beta 9 \text { ASCFMSZ }+\beta 10 \text { ASCDIST }+\beta 11 \text { ASCMEXP }
\end{aligned}
$$

\subsubsection{Marginal Willingness to Pay}

The marginal value of a change in an attribute could be given by the ratio of the coefficients of the attribute in question and that of the payment attribute, other things being the same. This is referred to as the part-worth or implicit price or marginal willingness to pay for the attribute. It represents the marginal rate of substitution between the payment attribute and the attribute in question.

$$
\mathrm{MWTP}=\frac{-\beta_{\text {attribute }}}{\beta_{\text {payment attribute }}}
$$

Moreover, the welfare changes from quality or quantity change of an environmental good (attributes) could be given by the measure of compensating surplus (CS) (Alpizar et al, 2003) and (Birol et al, 2005).

$$
\text { Compensating Surplus }=\frac{-(V o-V 1)}{\beta_{\text {payment attribute }}}
$$

Where, V0 represents the indirect utility at the status quo and $\mathrm{V} 1$ represents the indirect utility associated with environmental changes in various scenarios.

\section{Results and Discussion}

The questionnaire was divided into three blocks and six choice sets were provided for each of the 200 samples of respondents. Therefore, 1200 choices were elicited from this sample size and coded according to the level of the attributes. For the attributes of fish stock, at a high level improvement (limiting frequency of fishing to only once a month) was coded as 1 and the medium improvement (allowing fishing twice a month) was coded as 2 . For low level improvement and status quo a code 4 and 0 was given respectively.

For the attribute of biodiversity, a code 5, 10 and 20 were given to the level of planting trees on the $5 \mathrm{~m}, 10 \mathrm{~m}$ and $20 \mathrm{~m}$ wide land area along lake side. For availability of water attribute, accessibility of water in the four seasons of the year, three seasons of the year and two seasons of the year were coded as 4, 3 and 2 respectively.

The attributes of quality of water was coded as 5 and 10 for the reduction of run-off from the nearby industries by $50 \%$ (medium improvement) and 100\% (aggressive improvement). The levels for monetary payment attributes (i.e. 0, 50, 100, and 150 birr) were entered directly. The status quo alternative scenario levels were coded as 0 for all attributes. The data then entered in to NLOGIT5.0 software for analysis. The two multinomial logit models (basic and extended MNL models) and the Random Parameter Logit Model were estimated in the software. 


\subsection{Multinomial Logit Models}

Table 1. Results of the Basic Multinomial Logit model.

\begin{tabular}{llll}
\hline CHOICE & Coefficient & Standard Error & Prob. $|\mathbf{z}|>\mathbf{Z}^{*}$ \\
\hline ASC $\mid$ & $.78625^{* * *}$ & .25955 & .0025 \\
FST $\mid$ & -.05583 & .03741 & .1356 \\
BIOD $\mid$ & $.02244^{* * *}$ & .00660 & .0007 \\
WQ $\mid$ & $.06297^{* * *}$ & .01851 & .0007 \\
AOW $\mid$ & .06372 & .04713 & .1763 \\
PYNT $\mid$ & $-.0028^{* * *}$ & .00096 & .0036 \\
\hline
\end{tabular}

Note:***,***==>Significance at $1 \%, 5 \%, 10 \%$ level. R-sqrd .0196 R2Adj .0172

From the above table the result of estimated basic multinomial logit model revealed that the attribute of biodiversity and water quality has the expected sign and both are highly significant at $1 \%$ level of significance. This positive sign implies that an increase in the levels of these attributes increases the probability of choosing improved scenarios. In other words, the farmers (respondents) gave more weight for biodiversity and quality of water of the lake. The positive sign of the biodiversity attribute is consistent with results of other valuation studies (e.g., Birol et al., 2006; Despina, 2010). The attribute of water quality have also positive which is consistent with the previous studies (e.g., Nick et al. 2005). This implies that the residents around Lake Koka are highly affected by the pollution of the lake due to industrial run-off from the surrounding industries especially from Ethiopian tannery works which is highly polluting the Lake and wants the improvement of water quality of the Lake. Therefore respondents choose the highest level of water quality attribute improvement (total reduction of run-off $(100 \%))$. The sign of monetary attribute is negative as expected and significant at 1 percent level which is consistent with economic theory. This result indicates that, other things held constant, the higher the payment level in the alternative scenario, the less preferred it is by respondents.

The alternative specific constant has positive sign and it is significant which implies that there is welfare improvement as we move away from status quo.

Even though the expected sign for the fish stock is positive, it has negative sign, but it is insignificant. The attribute of availability have the expected positive, but insignificant at $1 \%$ level of significance.

\subsection{The Hausman-test of the IIA Assumption}

The IIA assumption states that the ratio of the probabilities of any two alternatives should be preserved despite the presence or absence of any other alternative within the set of alternatives included within the model.

Hausman and McFadden (1984) proposed a specification test for the MNL model to test the IIA assumption. The Hausman test is conducted by removing one or more alternatives from the choice set and compare the result of the test to the result of MNL model. When there is a change in the coefficients of the attributes (in magnitude and/or in sign), then the assumption of IIA is failed.

Table 2. The result of Hausman test for the IIA assumption.

\begin{tabular}{llllll}
\hline Choice & Coef & Standard error & Z & Prob. $|\mathbf{z}|>\mathbf{Z}^{*}$ \\
\hline ASC & $1.30256^{* *}$ & .61448 & 2.12 & .0340 \\
B_FST & -.06808 & .10552 & -.65 & .5188 \\
B_BIOD & .00711 & .01890 & .38 & .7067 \\
B_WQ & -.01195 & .04037 & -.30 & .7672 \\
B_AOW & .16059 & .11848 & 1.36 & .1753 \\
B_PYNT & -.00255 & .00250 & -1.02 & .3074 \\
\hline
\end{tabular}

Note: $* * *, * *, *==>$ Significance at $1 \%, 5 \%, 10 \%$ level.

The result of the above table reveals that when alternative one (alt 1) is removed from the choice set for the lake improvement, the coefficient of all attributes is changed both in magnitude and in sign.

As it can be observed from the table all attributes are insignificant as it opposes the result of the MNL model. This implies that the assumption of IIA cannot hold true as the choice probability of two or more alternatives are affected by the inclusion or exclusion of one or more alternatives in the choice set. For example, water quality attribute affect negatively the utility of respondents when alternative one is removed the choice set.

Random Parameter Logit Model

There are two problems with multinomial logit. The first problem is because of the assumption of the independence of irrelevant alternatives (IIA) will not hold true. The IIA property states that the ratio of the choice probabilities of any pair of alternatives is independent of the presence or absence of any other alternative in a choice set. A particularly important behavioral implication of IIA is that all pairs of alternatives are equally similar or dissimilar. Second problem is that, multinomial logit model does not take into account the taste variation or unobserved heterogeneity among individual respondents. Because of these two main problems of multinomial logit model, Random Parameter Logit model is used in order to incorporate the unobserved heterogeneity and to relax the IID assumption. Since the difference matrix was negative definite, the Hausman test is not conducted to find 
out whether the IIA assumption is violated in the multinomial logit model. However, a result of the random parameter logit model, which addresses these two problems with the multinomial logit model, is reported in table 2

Table 3. Results of Random Parameter Logit Model.

\begin{tabular}{llllll}
\hline CHOICE & Coefficient & Standard Error & $Z$ & Prob. $|z|>Z *$ & $95 \%$ Confidence Interval \\
\hline \multicolumn{7}{l}{ Random parameters in utility functions } \\
\hline B_FST & .08331 & .12703 & .66 & .5119 & -.16566 .33229 \\
B_BIOD & .03215 & .02732 & 1.18 & .2393 & -.02140 .08571 \\
B_WQ & .06146 & .07188 & .85 & .3926 & -.07943 .20235 \\
B_AOW & .24389 & .20743 & 1.18 & .2397 & -.16266 .65043 \\
\hline
\end{tabular}

Nonrandom parameters in utility functions

\begin{tabular}{llllll}
\hline ASC & $2.12123 * * *$ & .41023 & 5.17 & .0000 & 1.317202 .92527 \\
B_PYNT & $-.00249 * *$ & .00117 & -2.13 & .0328 & $-.00477-.00020$ \\
\hline \multicolumn{7}{l}{ Distns. of RPs. Std. Devs or limits of triangular } & & & \\
\hline NsB_FST & .02066 & .10353 & .20 & .8418 & .0002 \\
NsB_BIOD & $.05228 * * *$ & .01381 & 3.78 & .025225 .22357 \\
NsB_WQ & $.17255^{* * *}$ & 02962 & 5.83 & .0000 & .02520 .07935 \\
NsB_AOW & $.56634 * * *$ & .07845 & 7.22 & .0000 & .11450 .23060 \\
\hline
\end{tabular}

Note: $* * *, * *, *==>$ Significance at $1 \%, 5 \%, 10 \%$ level.

The $\mathrm{R}^{2}$ in the random parameter logit model is 0.2541 , while the corresponding value in the multinomial logit model is 0.0196 . Therefore there is improvement in the model fit with the use of random parameter model for the collected data set.

The interpretation of the output associated with the mean of a random parameter is in RPL model is the same as that of non-random parameter in the MNL model. For fish stock, biodiversity, water quality and availability of water the coefficient $0.08331,0.03215,0.06146$ and 0.24389 , are all insignificant implies that there is heterogeneity in preference of the respondents for these attributes. Therefore, the estimated constant parameter in MNL model for all respondents is insignificant.

In the last box the significance of the standard deviation of the parameters for attributes of biodiversity, water quality and availability of water suggests heterogeneity in preferences for those attributes.

\subsection{Estimation of Marginal Willingness to Pay}

The marginal willingness to pay or 'the implicit price' or 'the part worth' is the marginal rate of substitution between wetland attributes and the monetary attribute (Bennett and Blamely, 2001). It is the rate at which respondents are willing to pay for the improvement of an attribute. The value of the implicit prices of different attributes revealed the relative importance of the attributes for the society. The implicit prices are calculated as the ratio of the coefficients for the attributes of Lake Koka wetland ecosystem in random parameter logit model to the estimated coefficient of the monetary attribute. The results are reported in table 3 .

Table 4. Estimates of Marginal Willingness to Pay (in birr) for the attributes.

\begin{tabular}{lllllc}
\hline WaldFcns & MWTP in birr & Standard Error & Z & Prob. $|\mathbf{z}|>\mathbf{Z}^{*}$ & 95\%Confidence Interval \\
\hline Fst & -10.765 & 19.612 & -.55 & .5831 & -49.2060 \\
Biod & $12.1690^{* *}$ & 7.137 & 1.70 & .0882 & -1.8204 \\
Wq & $41.6678^{* * *}$ & 25.50 & 1.63 & .0102 & -8.3221 \\
Aow & $54.7331^{* * *}$ & 41.51 & 1.32 & .0187 & -26.1584 \\
\hline
\end{tabular}

Note: $* * *, * *, *=>$ Significance at $1 \%, 5 \%, 10 \%$ level.

From the result of table above, respondents are not willing to pay for the improvement of fish stock. This is because they assume that, limiting the amount of fishing will reduce their current consumption and lack of awareness about the benefit of fish attribute, but it is not statistically significant. However, the marginal willingness to pay for the improvement of water availability attribute is the highest which is birr 55 and it is significant at $1 \%$ level.

Respondent's willingness to pay for an increase in the level of water quality of Lake Koka and biodiversity from current situation of the lake, other things being constant, is birr 42 and birr 12 annually respectively. Respondents gave more value to the availability of water than biodiversity and water quality attributes.

\subsection{Estimation of Compensating Surplus}

One of the benefits of choice experiment method is that the estimated coefficients of the attributes can be used to estimate the economic welfare of the improvement of certain policy scenario. (Bergman et al, 2006). The results of the CE exercise can further be used to estimate the compensating surplus for a change from the initial situation (status quo) to different improved wetland management scenarios. 
It measures the change in income that would leave an individual indifferent between the initial (status quo) and subsequent situation (improved Wetland management) assuming that the individual has a right to the initial (status quo) level of utility. The difference between the utilities of the individuals that could be obtained from the status quo option and improved alternative scenarios is what economic welfare measures focus on.

Economic welfare measures or compensating surplus can be estimated by using the estimated coefficients of the attributes in the random parameter model (which is more explanatory relative to standard multinomial logit model) and the levels of the attributes in the different alternative scenarios It is estimated by using equation the following equation.

$\mathrm{CS}=\left(\frac{-(\mathrm{V} 0-\mathrm{Vi})}{\text { Bpynt }}\right)$, Where $\mathrm{V}_{\mathrm{i}}=\mathrm{V}_{1}$ and $\mathrm{V}_{2}$; and $\beta_{\text {pynt }}$ is the coefficient for monetary payment attribute. To calculate the economic welfare of the society, four hypothetical scenarios with their attribute levels were created. These are status quo, low impact improvement scenario, medium impact improvement scenario, and high impact improvement scenario.

Current situation/Status quo

1. No improvement in fish stock.

2. No improvement in biodiversity

3. No improvement in water quality

4. Water is available only in one season of the year (summer)

Low impact improvement scenario

1. Allowing fishing four time a month

2. Planting trees on the $5 \mathrm{~m}$ wide land area on the lake side

3. Reducing chemical run-off by $50 \%$

4. Water is available in two seasons of the month

Medium impact improvement scenario

1. Allowing fishing twice a month

2. Planting trees on the $10 \mathrm{~m}$ wide land area on the lake side

3. Reducing chemical run-off by $50 \%$

4. Water is available in three seasons of the month

High impact improvement scenario

1. Allowing once twice a month

2. Planting trees on the $20 \mathrm{~m}$ wide land area on the lake side

3. Reducing chemical run-off by $100 \%$

4. Water is available in all four seasons of the month

Table 5. Estimates of compensating surplus.

\begin{tabular}{ll}
\hline Improvement Scenario & Compensating surplus (in birr) \\
\hline Status quo & - \\
Low impact improvement & 162.57 \\
Medium impact improvement & 59.82 \\
High impact improvement & 175.46 \\
\hline
\end{tabular}

The estimated result revealed that, the respondent's willingness to pay (compensating surplus) increases as we improve the current situation of Lake Koka wetland ecosystem particularly the attributes of water quality, biodiversity and availability of water.

Respondents are willing to pay birr 175.46 , birr 59.82, and birr 162.57 annually for high impact, medium impact and low impact improvement scenarios respectively. The respondent's willingness to pay for the medium impact scenario is lower than that of for lower impact management scenario. This is due to differences in the level of fish stock attribute. When the level of improvement and management for fish stock improves from low to medium improvement scenario, it reduces willingness to pay of the respondent's from birr 165.57 to birr 59.82, which implies the respondent's unwillingness to pay for the improvement and conservation of fish stock as it will reduce their current consumption and income.

Moreover, the overall annual economic welfare (or the aggregate willingness to pay) so as to achieve, conserve and improve the stated attributes in the high impact scenarios, medium impact scenarios, low impact scenarios can be obtained by aggregating the mean willingness to pay for each scenario (birr 175.46, birr 59.82, and birr 162.57) into population.

Therefore, this result can be used as an input by the environmental policy makers, researchers, government, etc., to compute a cost benefit analysis for different alternative plan for the improvement of Lake Koka wetland ecosystem.

\section{Conclusion and Policy Recommendation}

\subsection{Conclusion}

Lake koka wetland ecosystem with an area of $250 \mathrm{~km}^{2}$ is located in the Misraq Shewa Zone of the Oromia Regional state, close to the capital and largest city of Ethiopia, Addis Ababa. It was created by the construction of the Koka Dam across the Awash River in 1966 for the purpose of electric power generation. The Lake is the backbone of the economy of the region in particular and the country in general. It has been serving the local peoples as source of drinking, animal watering, irrigation, recreation, cleaning, cultural contribution, fishing, etc.

Now a days, the lake is highly degraded due to its nature of public goods, and hence absence of enforceable property rights, open access to the wetland, lack of understanding about the values of wetlands, increasing population pressure, overgrazing, and failure of intervention by government. As a result many animal and plant species, bird species and fish species (a locally known as Bilcha and Koroso) were extinct. Businesses such as tanneries, flower farms, and manufacturing facilities of various things like soap and plastic products have set up along the banks and have virtually no regulations on how they get rid of their effluent. Because of this lack of regulation pollutants flow from the factories every day into the lake and the lake feeding it. This has been adversely influencing the nearby peoples, whose livelihoods depend up on presence and continuity of the lake and its ecological function. Therefore, something urgent needs to be done in order to curb the trend and create a convivial future.

The absence of studies on the valuation of the multidimensional benefits of wetlands of the lake which lowers the awareness of the society about the importance of 
these resources is the other problem related to the wetland. The study on welfare valuation of wetland of Lake Koka has not yet been undertaken. Therefore, this study fills this gap by applying choice experiment method. Hence, five attributes were identified namely, fish stock, biodiversity, water quality availability of water and monetary payment attribute.

Primary data was collected from a sample 200 respondents with six choice sets each and hence 1200 observations. Multinomial logit models, random parameter logit and latent class model were estimated by using NLOGIT5.0 econometric software. The result of the models revealed that the attributes of biodiversity and water quality are significant in affecting the probability of choosing an alternative scenario and have expected positive sign. However the attribute of availability of water has the expected positive sign but insignificant in MNL model and significant in the RPL model. On the other hand the attribute of fish stock has unexpected negative sign but it is also insignificant. The marginal willingness to pay for the improvement of water availability attribute is the highest which is birr 55 and it is significant at $1 \%$ level. Respondent's willingness to pay for an increase in the level of water quality of Lake Koka and biodiversity from current situation of the lake, other things being constant, is birr 42 and birr 12 annually respectively. This implies, respondents gave more value to the availability of water than biodiversity and water quality attributes.

Moreover, the estimated compensating surplus shows respondents are willing to pay birr 175.46, birr 59.82, and birr 162.57 annually for high impact, medium impact and low impact improvement scenarios respectively. This result confirms the residents need the improvement of the Lake wetland ecosystem and they are ready to pay for the improvement. Therefore, the government or any concerned body needs to take measures for the improvement of the lake wetland.

\subsection{Policy Recommendation}

Respondents (farmers) are willing to pay for the improvement the Lake Koka wetland ecosystem as the wetland is very important for their life and it is currently supporting a life of thousands of species (human, plant and different animal species). Therefore, the government or any concerned body can generate income from the farmers so as to improve, enhance and manage the Lake through improving the attributes of the Lake.

However, the results of the survey showed that respondents are willing to pay for the improvement of water quality and biodiversity attributes only. They are not willing to pay for the attribute of fish stock, because they expect that the designed policy for the improvement of fish stock through limiting frequency of fishing will affect their current consumption and income from the resource. Lack of awareness about the importance of the resource may also another reason which needs government and/or other voluntary organization to implement programme that create awareness about the importance of the resource.

The Lake could be the center of economy, culture, tourism and even national park when properly managed. Therefore, the Lake should be protected from different activities whether it is human made or natural that pollute and degrade the wetland. Moreover, the government should pay attention to the wetland and intervene in managing and controlling, introduce via different mass media like TV, radios, and newspapers etc. to improve the information of the society about the Lake. Furthermore, the industries built around the Lake like Ethiopian tannery Share Company should reduce chemical run-off to the Lake and have to highly participate in any programme designed to improve the quality of the Lake water. Additionally, government should also introduce a programme for the conservation of different species (fauna and flora) and bird species via planting tree species around the Lake.

\section{References}

[1] Adamowicz W. et al. (1994). Combining Revealed and Stated Preference Methods for Valuing Environmental Amenities. Journal of Environmental Economics and Management.

[2] Adamowicz, W., J. Louviere and J. Swait (1998). Introduction to Attribute-based Stated Choice Methods. NOAA-National Oceanic Atmospheric Administration Washington, USA.

[3] Ahmed s. (2014). Consumers' Willingness to Pay for Environmental Attributes of a Cut Flower in Ethiopia: A Choice Experiment Approach.

[4] Ajibola M. (2012). A Study of Wetland Valuation Practice for Compensation in The Niger Delta, Nigeria.

[5] Alpizar, F., F. Carlsson and P. Martinsson (2001). Using Choice Experiments for Non-Market Valuation; Working Paper in Economics no. 52, Department of Economics, Goteborg University.

[6] Ayenew. (2007). The changing face of the Ethiopian Rift-valley lakes and their environs.

[7] Bennett, J. and Blamey, R. (2001). The Choice Modeling Approach to Environmental Valuation New Horizons in Environmental Valuation,. Edward Elgar Publishing Limited, $U K$.

[8] Bennett J. et al. (2001). The Choice Modeling Approach to Environmental Valuation New Horizons in Environmental Valuation., Edward Elgar Publishing Limited, UK.

[9] Bergman, A., Hanley, N. and Wright, R. (2006) Valuing the Attributes of RenewableEnergy Investments. Energy Policy, 34 1004-1014.

[10] Birol E. et al. (2005). Using a Choice Experiment to Estimate the Non-Use Values of Wetlands: The case of Cheimaditida wetland in Greece: Environmental Economy and Policy Research Discussion Paper Series Department of Land Economy, University of Cambridge.

[11] Birol et al. (2008). Choice Experiments Informing Environmental Policy: A European Perspective. Munich: Munich Personal RePEc Archive.

[12] David A. et al. (2005). Appliedd choice analysis. New york: Cambridge University Press. 
[13] Edward B. (2013). Valuing Ecosystem Services for Coastal Wetland Protection and Restoration: Progress and Challenges:. Department of Economics and Finance, University of Wyoming 1000 E. University Ave., Laramie, WY 82071, USA.

[14] Egbenta (2010). Application of Contingent Method to Valuation of Non-Market Goods Damaged by Oil Pollution for Compensation.

[15] Finlayson, C. M., Davidson, N. C., Spiers, A. G., and Stevenson, N. J. (1999). Global Wetland Inventory: Current status and Future Priorities, Marine and Fresh water Research, $50,717-727$.

[16] Fitalew A. (2009). Valuation of Watershed and Fisheries of Lake Tana: An application of Choice Experiment; Unpublished Msc. Thesis, Addis Ababa University.

[17] Freeman, M. A. (1993), The Measurement of Environmental and Natural Resource Values. Theory and Methods, Resource for the Future, Washington, DC.

[18] G. V. T. Matthews. (2013). The Ramsar Convention on wetland its history and development. Ramsar Convention Bureau, Gland, Switzerland.

[19] Gashaw et al. (2015). stock assessment of fishery target species of lake Koka.

[20] Girma G. (2006). Valuing the Benefits of Improved Lake Quality: An Application of Choice Experiment to the case of Lake Awassa; Msc. Thesis, Addis Ababa University.

[21] Getnet. (2012). valuation of choke mountain wetland attributes. Addis Ababa University a Masters thesis.
[22] Hanley et al. (2001). Choice Modeling Approach: A superior Alternative for Environmental Valuation. Journal of Economic Surveys.

[23] Hanley, N., Wright, R., Adamowicz, V. (1998). Using Choice Experiments to Value the Environment: Design Issues, Current Experience and Future Prospects; Environmental and Resource Economics, 11 (3-4): 413-428. Kluwer Academic Publishers, Printed in Netherlands.

[24] International water management institute (2014).

[25] John A. (2009). Environmental valuation: challenges and practices; A personal view.

[26] Julian S. (2012) Comparing the Latent Class Model with the Random Parameters Logit - A Choice Experiment analysis of highly heterogeneous electricity consumers In Hyderabad, India. Department for Agricultural Economics, Humboldt-University.

[27] Lambert A. (2010). Economic Valuation of Wetlands: An Important Component of Wetland Management Strategies at the River Basin Scale.. A Discussion Paper of Ramsar Convention Bureau. Gland, Switzerland.

[28] Lancaster. (1966). A New Approach to Consumer Theory. Journal of Political Economy, Vol. 74 No. 2 pp. 132-157.

[29] Louviere, J. J., Hensher, D. A. and Swat, J. D. (2000) Stated Choice Methods: Analysis and Application. Cambridge University Press, Cambridge, Massachusetts, USA. 